\title{
A Simplified Model of Bit Error Rate Calculation
}

\author{
Saed Thuneibat ${ }^{1}$, Huthaifa Al Issa ${ }^{1} \&$ Abdallah Ijjeh ${ }^{1}$ \\ ${ }^{1}$ Department of Electrical Engineering, Al-Balqa Applied University, Jordan \\ Correspondence: Saed Thuneibat, Department of Electrical Engineering, Al-Balqa Applied University, Jordan. \\ E-mail: Thuneibat@hotmail.com/alissahu@yahoo.com/dr.a.ijjeh@gmail.com
}

Received: October 11, 2015

doi:10.5539/cis.v9n1p41

\author{
Accepted: October 16, $2015 \quad$ Online Published: December 20, 2015 \\ URL: http://dx.doi.org/10.5539/cis.v9n1p41
}

\begin{abstract}
The Bit Error Rate (BER) is a key parameter of the Quality of Service (QoS) for engineers and designers of digital communication systems and networks. At the present time, a set of models and methods are exist for calculating the BER. But these methods are complex and require large computing cost.

In this paper, we provide a new model for calculating the BER. This model simplifies the procedures in the existing models and reduces the computing time. In the same time, the proposed model save the accuracy and the state consideration of existing models.
\end{abstract}

Keywords: Bit Error Rate, Quality of Service, Modeling, Communication System

\section{Introduction}

Many countries have built or developed their local and national telecommunication networks. Nowadays, the issue is in providing the adequate QoS parameters of the data transmission and delivery to the end user.

Real-world communication channels experience noise and distortions. If we represent noise and Intersymbol Interference (ISI) on Euclidian space with power and time axis, then the noise internal and external, impulsive and induced, cross talk and attenuation are represented by power axis. Distortion, fading channel, phase distortion, delays, jitters and ISI are reflected by time axis.

Noise and ISI cause errors in a digital communication channels. Error, in a digital communication system is a condition when the transmitted bit is 1 , while the received is 0 , and vice versa.

In digital transmission, BER is the percentage of bits that have errors relative to the total number of bits received in one connection or session of communication. Usually, an electrical transmission of data might have a BER of $10^{-6}$, meaning that, out of 1,000,000 bits transmitted, one bit was in error. While an optical system might achieve $10^{-12}$. The BER is an indication of how often data has to be retransmitted because of an error, using one of the Automatic Repeat request (ARQ) methods (Jahangir, 2011). Alternatively, errors might be corrected without retransmission by applying channel coding schemes and redundant forward error correction codes such as Hamming codes (Thuneibat, 2012).

Digital communication channel encounters the errors in a bursty fashion. This means that in the transmitted frame of data, a group of bits, not single bit, had changed. The reason of such behaviour is found in the serial transmission mode and in the nature of random noise. The error control techniques are function of the data link layer of the Open System Interaction (OSI) reference model.

In order to design effective communication systems for such channels, it is critical to fully understand their behaviour and define an estimation equation for there calculation. This is achieved via system modelling, where the primary objective is to provide a model whose properties are both complex enough to closely, as possible, match the real system statistical characteristics, and simple enough to allow software simulation system analysis.

Providing guaranteed BER, data rates, delays and other indicators of subscriber loyalty requires the search for new solutions to make full use of the bandwidth. To compare the effectiveness of the decisions, simulation is implemented effectively. Modeling involves the selecting of a channel model; development of models linking qualitative indicators with the internal parameters of systems and channels; development of techniques to optimize the internal parameters to achieve the required transmission quality indicators.

The BER is the key of QoS parameter for the engineers and designers of digital communication systems and 
networks. At the present time, a set of models and methods are exist for calculating BER, but these methods are complex and require large computing cost.

In this paper, we discuss the calculation methods of ${ }_{P(m, n)}$, where ${ }_{P(m, n)}$ is the probability of $m$ errors in the received $n$ frames of data elements (bits) for the channels described by Gilbert and Gilbert-Elliot models (Elliott, 1963). Definition of $P(m, n)$ is necessary for the analysis and optimization of data transmission system. Therefore, our research is become more significant.

A new model for calculating the BER is proposed in this paper, where this model simplifies the existing procedures and reduces the computing time. In the same time, the proposed model save the accuracy and the state consideration of existing models.

\section{Exploring the Existing Models}

Gilbert-Elliott channel model is a two-state Markov channel, each state satisfies a communication channel quality, one state for noiseless, while the other is very noisy. The crossover probabilities for each state are 0 and 0.5 , respectively.

According to the existing models under considerations, implementing the Gilbert-Elliott model, which is a simple channel model introduced by Edgar Gilbert and E. O. Elliott (Elliott, 1963). These models are widely used for mapping burst error patterns in a digital transmission channels, that enables simulations of the digital error performance of communication links (Hong, 1995). It is based on a Markov chain with two states $G$ (for good or gap) and $B$ (for bad or burst), where in state $G$, the probability of transmitting a bit correctly is $k$, and in state $B$ the probability is $h$. Usually, it is assumed that $k=1$ and Gilbert also assumed that $h=0.5$ (Gilbert, 1960).

The BER in states $G$ and $B$ is different, so before we calculate $P(m, n)$, we determine the probability of $i$ bits where transferred in the bad state of channel from $n$ frames $B(i, n)$. Then the probability of the $m$ errors in $n$ frames of bits can be determined using the following expression

$$
P(m, n)=\sum_{i=0}^{n} B(i, n) P(m / i),
$$

where

$$
P(m / i)=C_{i}^{m} p_{\text {error }}^{m}\left(1-p_{\text {error }}\right)^{i-m} .
$$

According to Gilbert model, $P(m / i)$ is the conditional probability of $m$ errors in $_{i}$ bits transmitted in the $B$ state, while $p_{\text {error }}$ is the probability of errors in the $B$ state and $C_{i}^{m}$ is number of combinations of $i$ from $m$.

Major accounted cost when calculating $B(i, n)$ is spent on determining the probability $B(i, n)$.

Covariance matrix method is proposed for the exact solution of this issue, which reduces the requirements to computing resources in the calculation of $B(i, n)$ (Holzlöhner, 2003).

The modeling of communication system as two-state Gilbert-Elliott channel is not adequate when the channel quality varies dramatically. The solution is to design a model with more than two states.

In this paper, we add a new state for reflecting a combination of the physical condition of the channel $S_{j}$ and the combination state denoted as $S_{j}(k, l)$, as shown in figure 1 . 


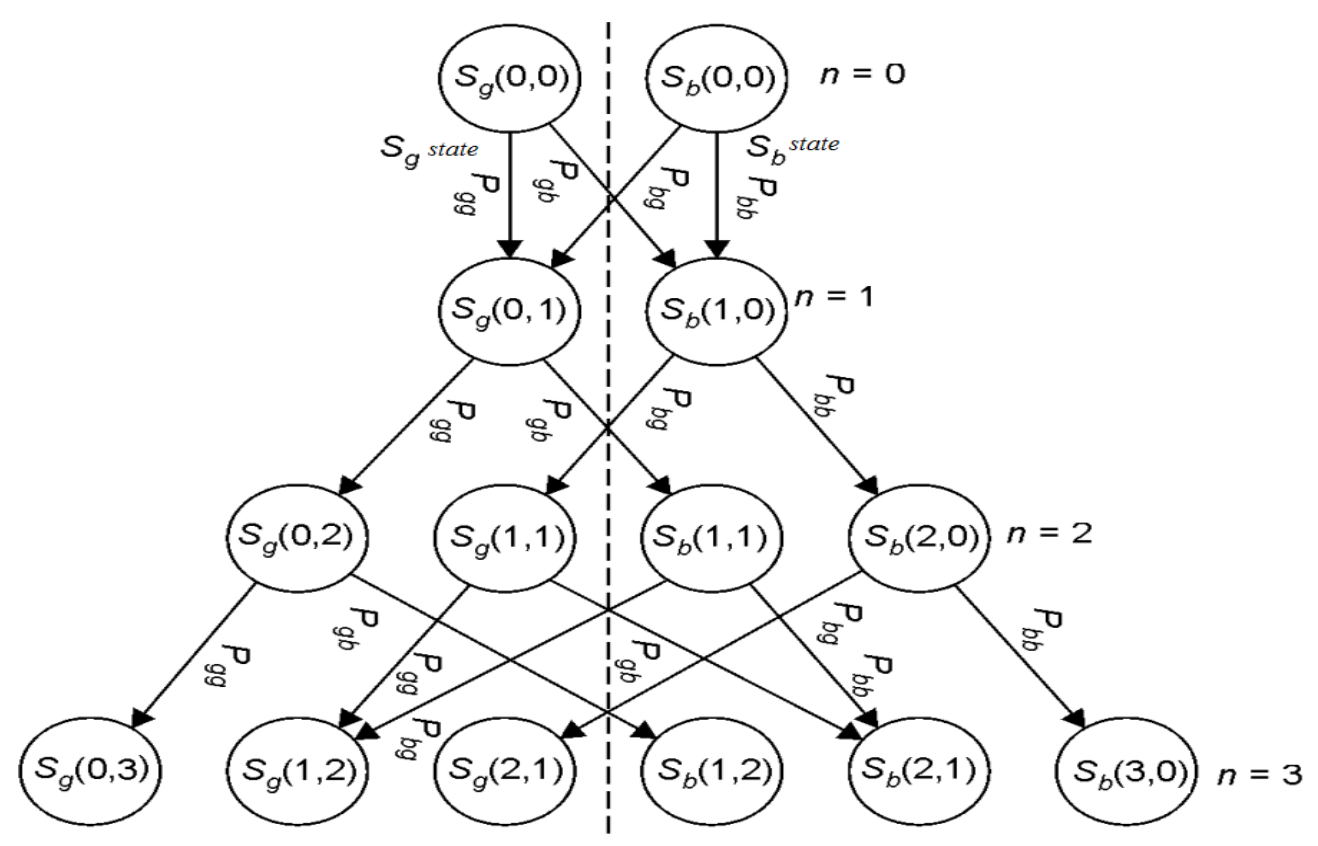

Figure 1. State graph of a system with combined states

In the figure, $n$ is the number of hops of existence in the introduced state, $k$ is the number of hops of existence in $S_{b}$ state, and $l$ is the number of hops of existence in $S_{g}$ state, where $k+l=n$.

The initial states when $n=0$ are represented by $S_{b}(0,0)$ and $S_{g}(0,0)$ respectively. Let the initial probabilities to be $p_{b}$ and $p_{g}$, the initial probability vector through $\| \begin{array}{ll}p_{b} & p_{g} \|\end{array}$ while $_{p_{b}+p_{g}=1}$.

The developed structure increases the number of states at each hop. Thus, at each hop we increase the transition matrix dimension and the system state vector.

In the first transition hop, the probability matrix satisfies the original matrix of Gilbert models. The transition probability matrix of the $n$-th step has a dimension of $2(n-1) \times 2 n$; as follow:

$$
\mathbf{A}(n)=\left\|\begin{array}{ll}
A_{b b} \mid \dot{0} & A_{b g} \mid \dot{0} \\
\dot{0} \mid A_{g b} & \dot{0} \mid A_{g g}
\end{array}\right\|
$$

where $A_{b b} \mid \dot{0}$ is a diagonal matrix, extended from the right side by one zero column; $\dot{0} \mid A_{g b}$ is diagonal matrix, extended from the left side by one zero column; $A_{\mathrm{bb}}, A_{\mathrm{bg}}, A_{\mathrm{gb}}, A_{\mathrm{gg}}$ are diagonal matrixes of (n-1) order, the nonzero elements are equal to $P_{b b}, P_{b g}, P_{g b}, P_{g g} ; \dot{0}$ is a column of zeros, expanding the matrix to the right or left.

The above explained approach provides a distribution vector of the probabilities for the combined state at any hop $\vec{b}(n)$. For the calculation of $B(i, n)$ values, the summation probability of states $b_{j}(k, l)$ is taken having the same first index. Thus, the scheme of forming probabilities $B(i, 3)$ as example has the form as shown in figure 2 .

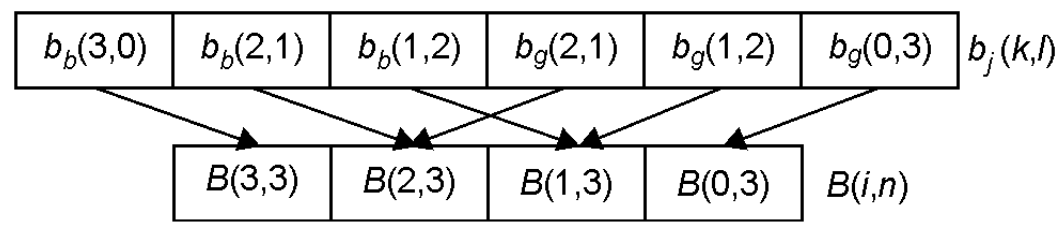

Figure 2. The scheme of forming $\mathrm{B}(\mathrm{i}, 3)$ 
As will these probabilities may be written in the form of:

$B(3,3)=b_{b}(3,0) ;$

$B(2,3)=b_{b}(2,1)+b_{g}(2,1) ;$

$B(1,3)=b_{b}(1,2)+b_{g}(1,2)$;

$B(0,3)=b_{g}(0,3) \cdot$

In general we write:

$B(n, n)=b_{b}(n, 0)$;

$B(0, n)=b_{g}(0, n)$;

$B(i, n)=b_{b}(i, n-i)+b_{g}(i, n-i)$

When the packet of data has the length of hundreds of bits, which is the situation faced in the Internet, the packet size may reach 65336 bytes of data, then the required computing time increase notably. This situation may reduce the effectiveness of the application of technique in adaptive data transmission systems. For significant time saving, we show in the next section, the proposed simplified method of calculation.

\section{The Proposed Model}

We introduce the concept of the channel state vector as an array of binary numbers, where zero corresponds to the transfer of the current bit to good state, and one - the transfer of the current bit to bad state of channel. We notify the number of bad state sections of the channel on the length of the frame as the number of returned bad state.

The required value $B(i, n)$ is the sum of the probabilities of all state vectors of the channel with length $n$ and weight $i$.

Unlike channels with independent errors, in channels with dominant burst errors, the probability of vectors of the same weight will depend on the number of returns to the bad states of the length of the vector. Probability vectors with frequent alternation between 0 and 1 (i.e., multiple transitions from good to bad states) may have smaller probabilities than the vectors of the same weight, but with a small number of returns to bad states.

By excluding from the calculation the vectors, reflecting the multiple return of $B$-state, as show in figure 3, we can significantly reduce the time of calculation, while maintaining an acceptable accuracy level.

Naturally, such an approach would give some uncertainty of the result, depending on the number of accounted returns, the length of the frame and the probability of changing the states of channel. The criterion for the estimation of the uncertainty introduced by discarding terms that take into account multiple returns, can be written as

$$
\delta=1-\sum_{i=0}^{n} B(i, n)
$$

The expressions that take into account the contribution of vectors with one, two and $v$ returns to the probability of $B(i, n)$ are written below

$$
C_{i-1}^{\nu-1} \cdot P_{b} \cdot P_{b b}^{i-v} \cdot P_{b g}^{v-1} \cdot P_{g g}^{n-i-v-1} \cdot P_{g b}^{\nu-1}\left[C_{n-i-1}^{v-2} \cdot P_{g g}^{2}+2 C_{n-i-1}^{\nu-1} \cdot P_{b g} \cdot P_{g g}+C_{n-i-1}^{v} \cdot P_{b g}^{2}\right]
$$

for $v \leq i \leq n-v-1$;

$$
C_{i-1}^{v-1} \cdot P_{b} \cdot P_{b b}^{i-v} \cdot P_{b g}^{v-1} \cdot P_{g g}^{n-i-v} \cdot P_{g b}^{v-1}\left[C_{n-i-1}^{v-2} \cdot P_{g g}^{2}+2 C_{n-i-1}^{v-1} \cdot P_{b g}\right]
$$

for $i=n-v$;

$$
C_{i-1}^{v-1} \cdot P_{b} \cdot P_{b b}^{i-v} \cdot P_{b g}^{v-1} \cdot P_{g g}^{n-i-v+1} \cdot P_{g b}^{v-1} \cdot C_{n-i-1}^{v-2}
$$

for $\quad i=n-v+1$.

These expressions are valid for the number of returns from the $v=3$ and higher. 


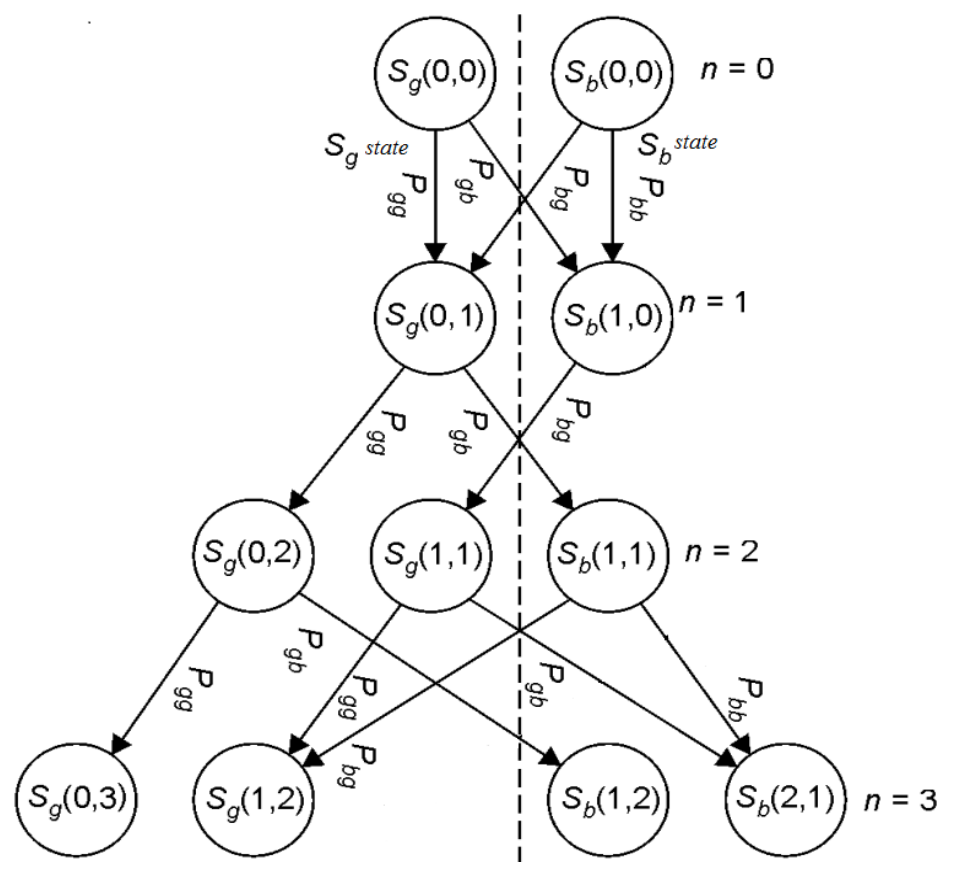

Figure 3. Simplified state graph of model

\section{Evaluation of the Simplified Model}

Further, we evaluate the uncertainty of results in simplified methodology relative to the values obtained by the exact algorithm, using the following expression

$$
\Delta_{b}=\frac{P_{E}-P_{S}}{P_{E}} \cdot 100
$$

where $P_{E}, P_{S}$ the values obtained by exact algorithm and simplified methodology, respectively.

The dependence of the relative difference $\Delta_{b}$ of calculation results $B(i, n)$, obtained by the use of accurate and simplified methods when the number of returns $v$ equals $2,3,4$ and 5 are shown in figure 4 .

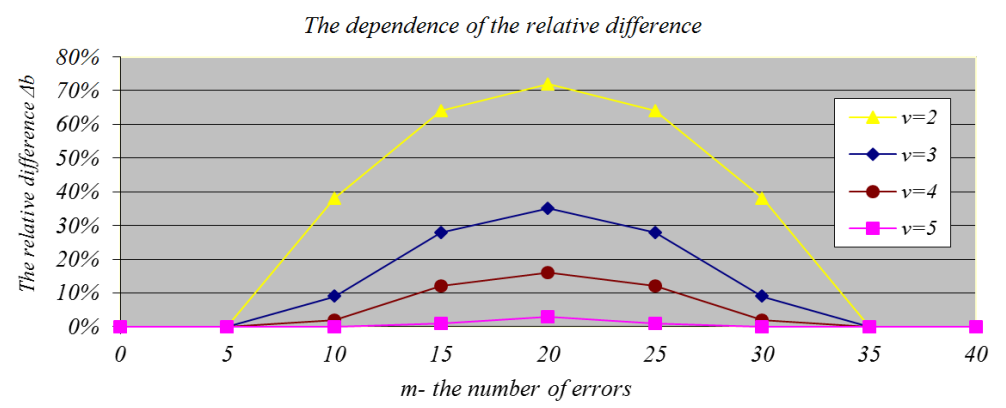

Figure 4. The dependence of the relative difference

From the figure, we see that the maximum error observed near the middle of the frame and noticeably reduced by increasing the number of returns. Practically, we are interested in the error probability, which is much less than half the length of the data packet, and in this area the uncertainty is much less than the maximum, which also justifies the use of the simplified model.

For the comparison of calculation costs of different models, we select the parameters that quantifiably characterizing these costs and not dependent on the speed of the processor or the algorithm. 
We choose parameters such as the number of additions and multiplications required to compute the value of probability $P(m, n)$ and perform the calculation.

Figure 5 shows the dependence of the number of operations of and multiplication in the computation of $P(5, n)$ for $\mathrm{n}$, ranges from 5 to 100 in the calculations by the Elliott method, and a simplified methodology if the number of returns equals 7 .

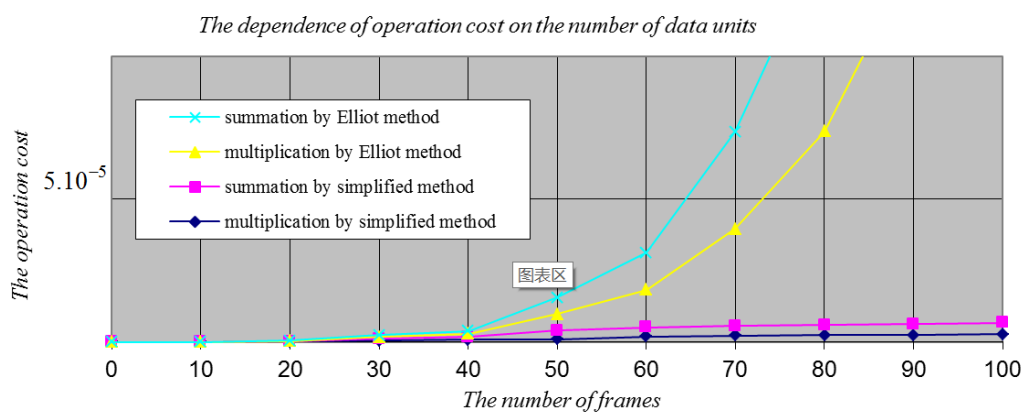

Figure 5. The dependence of the operations cost in calculating $\mathrm{P}(5, \mathrm{n})$

The curves in figure 5 clearly show the advantage of the simplified method. The operation cost increases exponentially for data length greater than 40 frames for both summation and multiplication operations by Elliot method. While the operation cost remains approximately constant using the proposed simplified method.

\section{Conclusion}

The matrix method of calculating the exact probabilities of burst error in data packet of fixed length for communication channel described by Gilbert model is explained. The new simplified model, which proposed in this paper, provides a significant reduction in the computing cost while maintaining acceptable accuracy level.

\section{References}

Elliott E. N. (1963). Estimates of error rates for codes on burst-noise channels (pp. 1977-1997). Bell Syst. Tech. J., 42.

Gilbert, E. N. (1960). Capacity of a burst-noise channel, burst-noise channel. Bell Syst. Tech. J., 39.

Holzlöhner, R., Menyuk, C. R., Kath, W. L., \& Grigoryan, V. S. (2003). A covariance matrix method to compute bit error rates in a highly nonlinear dispersion managed solution system (pp. 688-690). IEEE Photon. Technol. Lett., 15.

Hong, S. W., \& Nader, M. (1995). Finite-State Markovf Channel-A Useful Model for Radio Communication Channels (pp. 1253-1265). IEEE Transaction On Vehicular Technology, 44(1).

Jahangir, A., Alam, M. R., Hu, G. Q., \& Mehrab, M. Z. (2011). Bit Error Rate Optimization in Fiber Optic Communications (pp. 435-440). International Journal of Machine Learning and Computing, 1(5).

Thuneibat S. A. (2012). 3-D Hamming Code for Burst Error Correction Over Wireless Communication Channel (pp. 80-85). International Journal on Communications Antenna \& Propagation, 2(1).

\section{Copyrights}

Copyright for this article is retained by the author(s), with first publication rights granted to the journal.

This is an open-access article distributed under the terms and conditions of the Creative Commons Attribution license (http://creativecommons.org/licenses/by/3.0/). 